

$\begin{array}{llll}\Omega & \square & \equiv & \text { @ } \\ \text { P.M. Putora, } & \text { Kantonspital St Gallen, } & \text { P. M. Putora Klinik für } & \text { paul.putora@kssg.ch } \\ \text { T. Schneider, } & \text { St Gallen, Switzerland } & \text { Rodio-Onkologie, } & \\ \text { R. Rodriguez, } & & \text { Kantonspital St Gallen, } \\ \text { M. Früh } & & \text { Rorschacherstrasse 95, } \\ & & \begin{array}{l}\text { Haus O3, St Gallen 9007, } \\ \text { Switzerland }\end{array}\end{array}$

\section{Targeted therapy in non-small cell lung cancer}

\section{Educational aims}

- To explain the clinically relevant molecular pathways in lung cancer.

๑ To understand the basic principles of tests useful for targeted therapies.

- To present a basis for current treatment approaches.

\section{Introduction}

The treatment outcomes in lung cancer continue to be disappointing. Even with optimal combinations of surgery, radiotherapy and chemotherapy, less than $15 \%$ of patients can be cured. Chemotherapy is routinely used in most lung cancer patients and local therapies (e.g. surgery, radiotherapy) are standard options in early and locally advanced disease. Decades of intensive research have led to the identification of molecular pathways which are of central importance in cancer development and tumour growth. Drugs have been developed to target these mechanisms in order to improve outcomes of lung cancer patients. Fortunately, several targeted agents have recently been established in the treatment of lung cancer and will be discussed.

\section{Lung cancer}

Following the industrial production of cigarettes and the subsequent rise of tobacco consumption, the lung cancer epidemic rose. The last few years have shown some hope with a reduction in cancer-related deaths, not least due to the tobacco cessation campaigns [1]. Today, lung cancer is still the leading cause of cancerrelated death with an estimated 1,600,000 new cases and 1,380,000 deaths worldwide in 2008 [2]. Symptoms such as cough, weight loss, dyspnoea, chest pain, haemoptysis or bone pain and hoarseness are often results of locally advanced or metastatic disease [3]. Three out of four symptomatic patients have advanced stage and not amenable to cure [4].

The optimal diagnosis and therapy is only possible when the exact histology is provided and certain molecular markers examined. In order to obtain the required samples, different techniques are used depending on cancer extent and location. Image-guided (computed tomography or ultrasound) percutaneous needle aspiration or biopsy is feasible when lesions are located near the pleura. The accuracy drops and the risk of pneumothorax rises with increasing distance from the pleura [5]. Flexible bronchoscopy has the advantage of visualising
HERMES syllabus link: module B. 2. 1 
endobronchial spread and enables forceps biopsy or/and transbronchial fine needle aspiration. The sample yield in peripheral cancers can be improved with the use of endobronchial ultrasound used during fine needle aspiration, brush or biopsy. The use of navigational tools may further improve results [6]. For invisible central cancer due to submucosal growth or for mediastinal staging, the endobronchial ultrasound technique opens a new era of minimally invasive diagnostic yield [7]. When imaging is positive and sampling negative, surgical procedures such as mediastinoscopy or thoracoscopic wedge resection may be required to allow for a diagnosis and secure proper staging. The quality of the tissue obtained by these procedures is of crucial importance for subsequent histological and molecular diagnosis, which is a centrepiece of targeted therapy.

\section{Molecular pathways}

The molecular pathways involved in cancer development, cancer cell proliferation and treatment resistance are very complex. Currently only a few of numerous identified molecular targets are relevant in clinical practice outside of clinical trials. The most important targeted treatment options in lung cancer currently include the epidermal growth factor receptor (EGFR), the vascular endothelial growth factor receptor (VEGFR) and the echinoderm microtubuleassociated protein-like 4-anaplastic lymphoma kinase fusion gene (EML4-ALK).

\section{EGFR}

The ErbB family of receptors includes the four members: ErbB-1 (EGFR), ErbB-2 (HER-2) which is relevant especially in breast cancer treatment, ErbB-3 and ErbB-4. The extracellular component is responsible for ligand binding; whereas the intracellular component consists of the tyrosine kinase, which is responsible for signal transduction.

After a ligand (e.g. epidermal growth factor, transforming growth factor- $\alpha$ ) binds to the extracellular receptor, a dimerisation results in the activation of the receptor. The signal is passed along various downstream pathways. The most relevant seem to be the RAS/RAF/ MEK/MAPK pathway, the PI3K/AKT pathway and the STAT3/STAT5 pathway [8]. These pathways lead to changes in gene transcription and alterations in the cell cycle, resulting in

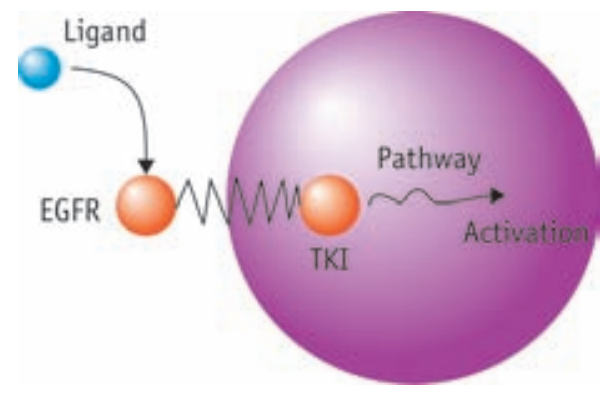

Figure 1

Epidermal growth factor receptor (EGFR), the intracellular tyrosine kinase (TKI) and the following intracellular pathways.

increased cell proliferation and angiogenesis, inhibition of apoptosis and changes in their capacity for migration, adhesion and invasion. As one would expect, changes in these parameters and properties have an impact on the behaviour of the cancer.

There are various mutations that have been discovered in the EGFR gene. Some of these mutations lead to an increased activity of the tyrosine kinase and subsequent sensitivity to targeted therapy whereas others increase cell resistance to treatment with tyrosine kinase inhibitors (TKIs).

\section{VEGF receptor}

After ligand binding, proliferation and migration of endothelial cells are activated, leading to increased blood supply of tumour cells and contributing to cancer cell survival [9].

\section{EML4-ALK}

This oncogene is created by an inversion on chromosome $2 \mathrm{p}$. In this mutation the $\mathrm{N}$ terminal end of EML4 fuses with the intracellular domain of ALK. Breaking points in EML4 vary, whereas they seem to be constant in ALK,

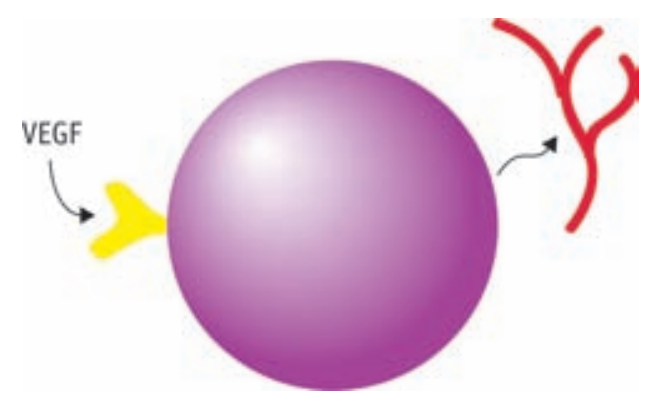

Figure 2

Vascular endothelial growth factor (VEGF) can bind to the appropriate receptor leading to vascular proliferation. 


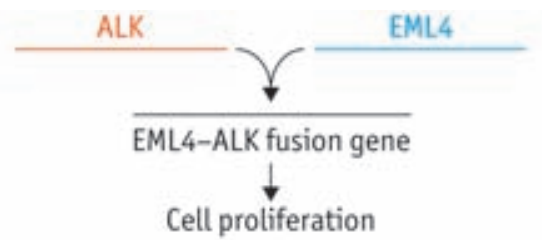

Figure 3

The fusion of the EML4 and ALK genes leads to a fusion oncogene.

several fusion variants have been discovered. The fusion leads to a constitutive activation of a chimeric tyrosine kinase leading to increased cell proliferation [10, 11].

\section{Testing}

The recipe for proteins is coded in genes, these are represented by the DNA. Before a gene is expressed, it is transcribed to mRNA, which then is translated to the protein.

There are several tests that can be performed on different levels from DNA segments to proteins. The tests that have been established and are relevant for treatment decisions include PCR-DNA sequencing, fluorescence in situ hybridisation (FISH) and immunohistochemistry (IHC).

\section{PCR and DNA sequencing}

Mutations within a gene may lead to a change in the functioning of the resulting protein. An identified gene is amplified (multiplied) by PCR before it can be sequenced. With DNA sequencing specific variants (mutations) of genes may be identified. Depending on the gene and the mutation these may lead to reduced or even increased function.

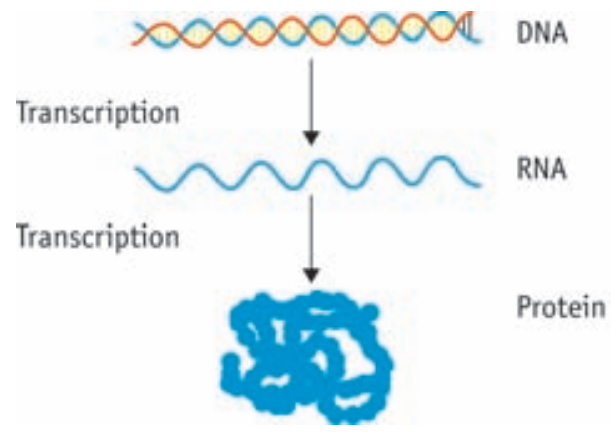

Figure 4

DNA is transcribed to RNA, this can then be translated to proteins.
Sequencing nucleotide by nucleotide has been made possible by new technologies. With increased capacities and capabilities, sequencing has become available outside research settings.

With gene sequencing, various changes in the DNA sequence can be identified; these include insertions, deletions or point mutations. The changes in sequence lead to changes in transcription to amino acids, thereby changing the form of a protein.

The advantage of gene sequencing is that specific mutations can be identified. Currently, many EGFR mutations have been identified. Many of those have shown to be associated with good responses to EGFR inhibiting drugs (e.g. deletion in exon 19 or point mutation in exon 21 L858R), some however have shown to be associated with resistance (e.g. exon 20 T790M).

Kirsten rat sarcoma viral oncogene homologue (KRAS); activating KRAS mutation have demonstrated to be associated with resistance to EGFR inhibitors. KRAS and EGFR mutations are considered to be mutually exclusive in lung cancer patients [12]. In practice, however, both tests are usually performed for control.

\section{FISH}

FISH is a technique that allows the visualisation of specific chromosome nucleic acid sequences (genes) within a cellular preparation. Specifically, FISH involves the precise annealing of a single-stranded, fluorophore-labelled DNA probe to complementary target sequences. After preparation, the DNA of the tissue on slides is denatured to single-strands and hybridised with DNA probes. Following hybridisation, the unbound probe is removed by a series of washes. The flurophore-labelled probes, now bound to the cellular DNA, can be visualised in fluorescence microscopy.

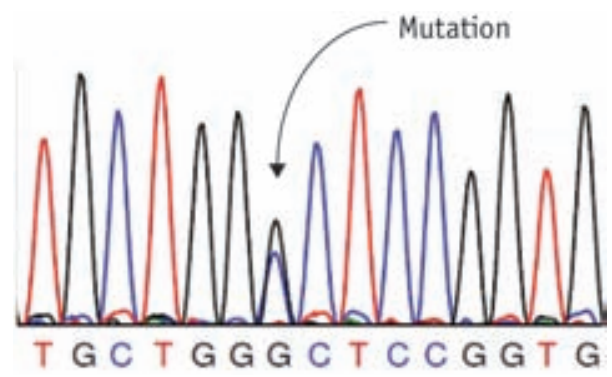

Figure 5

With the help of DNA sequencing, variations can be identified. 


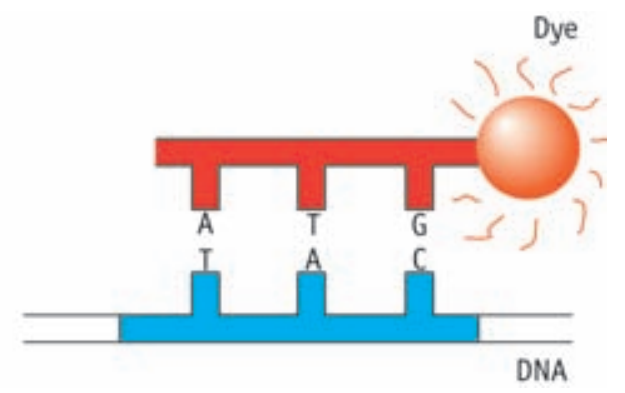

Figure 6

A complementary DNA is labelled with a fluorescent dye and allowed to bind to a specific sequence on the DNA.

To detect EML4-ALK fusion genes, fluorescence in situ hybridisation can be used. When hybridised with the appropriate labelled FISH probes, the 2 p23 ALK region in its native state will be seen as two immediately adjacent or fused (overlapping) orange/green (yellow) signals. However, if a chromosome rearrangement at the 2p23 ALK breakpoint region has occurred (inversion), one red and one green signal separated by at least two signal diameters will be seen.

\section{IHC}

With specific dyes, antibodies to proteins can be labelled and can then be visualised under the microscope.

Immunohistochemistry refers to the process of detecting proteins by binding labelled

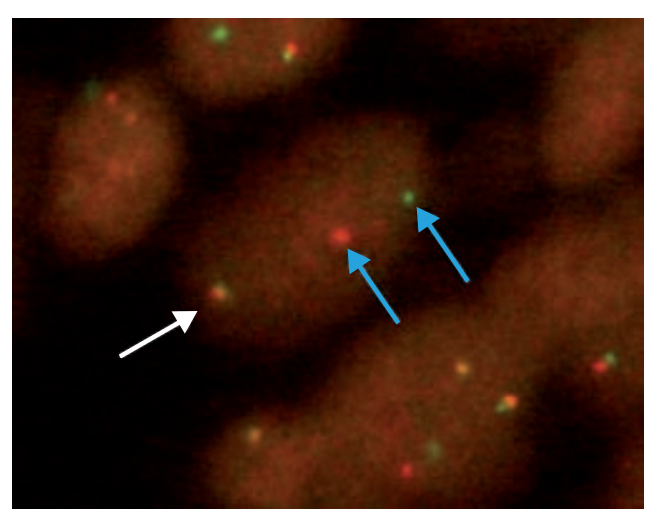

Figure 7

The fluorescence in situ hybridisation image shows ALK gene (red represents the telomer side of the ALK gene, whereas green represents the centromere side). The white arrow shows the genes in the same location (wild-type), the blue arrows show separated signals after mutation (inversion) of the gene.

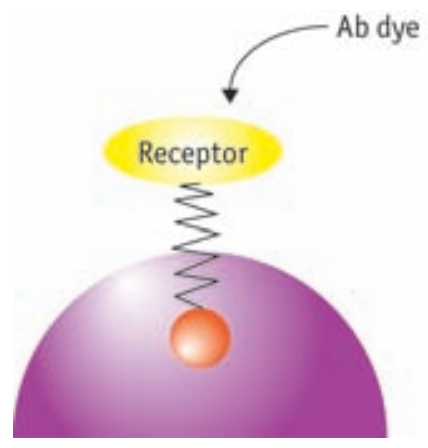

Figure 8

Immunohistochemistry; with the help of labelled antibodies $(A b)$ the expression of receptors and proteins can be visualised.

antibodies to these. Visualising an antibodyantigen interaction can be accomplished in a number of ways. In the most common instance, an antibody is conjugated to an enzyme, such as peroxidase, that can catalyse a colourproducing reaction.

IHC methods have been successfully used to evaluate the expression (over-expression) of the EGFR, which is of clinical relevance for EGFR antibody therapy.

\section{Principles of treatment}

\section{EGFR inhibition}

With the identification of receptors and pathways playing important roles in cancer, drugs have been developed to influence them. In the case of the EGFR, drugs have been developed to inhibit the transfer of the activating signal to intracellular pathways. These include agents which can block the receptor from outside the cell (antibodies) or drugs acting intracellularly (tyrosine kinase inhibitors (TKIs)).

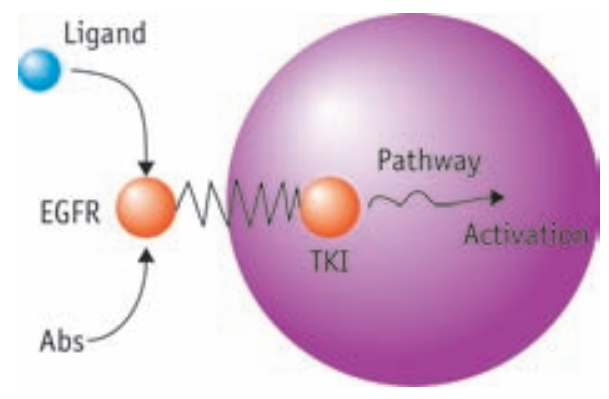

Figure 9

Antibodies and tyrosine kinase inhibitors (TKI) can inhibit signal transduction from the extracellular environment to intracellular pathways. Ab: antibody; EGFR: epidermal growth factor receptor. 


\section{VEGF inhibition}

Antibodies specific for the VEGF Receptor can inhibit the downstream signalling leading to inhibition of angio- and vasculogenesis. The principle is analogous to the antibodies in EGFR inhibition. In addition, numerous small molecule tyrosine kinase inhibitors, including sunitib, sorafenib, vandetanib and others, have been explored.

\section{EML4-ALK inhibition}

The EML4-ALK fusion oncogene is responsible for cell proliferation. With the help of a targeted agent against the ALK intracellular tyrosine kinase, the function of the tyrosine kinase can be inhibited, thus blocking further downstream signalling. This is analogous to the functioning of the TKIs in the aforementioned EGFR example.

\section{Resistance}

Although the principles of treatment seem very clear and effective, the clinical activity is limited by the development of resistance to these agents. For EGFR tyrosine kinase inhibitors (erlotinib, gefitinib) secondary mutations or bypass activation of alternate pathways, which may lead to a downstream signalling, lead to resistance. These mechanisms have been specifically described and represent a significant proportion of resistance development [13, 14]. With other targeted treatments, similar mechanisms have been described, e.g. for crizotinib and the EML4-ALK fusion oncogene [10].

\section{Clinical results}

\section{EGFR TKIs}

Patients whose tumours harbour an activating EGFR mutation (e.g. deletion in exon 19 or point mutation in exon 21 (L858R)) derive significant benefit from the treatment with small molecule TKIs. The first trial to demonstrate, that the first-line use of a TKI is superior to chemotherapy in these patients was a subgroup analysis of the IPASS study which enrolled $>1,200$ Asian patients and compared carboplatin/paclitaxel with gefitinib in patients with adenocarcinoma who were light or never smokers [15]. Since this report, several prospective randomised phase-III studies in patients with EGFR mutations confirmed these findings, demonstrating a progression free survival of about 1 year and survival rates of up to 28 months [16-18]. Based on these results, EGFR TKIs (e.g. erlotinib, gefitinib) today represent the preferred initial systemic treatment option for patients with an activating EGFR mutation.

\section{EGFR antibodies}

Cetuximab is an inhibitory anti-EGFR antibody which interacts with domain III of the soluble extracellular region of EGFR, sterically preventing the receptor from adopting the extended conformation required for dimerisation and ultimately intracellular pathway activation. In the phase-III FLEX trial, cetuximab improved response rate and overall survival when added to cisplatin/vinorelbine in patients with advanced NSCLC expressing EGFR by immunohistochemistry. Response rates were statistically significantly improved from 29 to $36 \%$; however, the median survival was only improved from 10.1 to 11.3 months [19]. As the survival benefit was relatively small, approval was denied by health authorities. Reports have shown that a high IHCEGFR score (i.e. $>200$ ) can be used to identify about a third of patients in whom the addition of cetuximab to chemotherapy was associated with an increased benefit [19].

\section{Anti-angiogenic treatment}

Bevacizumab is a recombinant humanised monoclonal antibody that binds to and neutralises the biological activity of human VEGF [20]. This agent was the first targeted treatment showing a survival advantage in lung cancer [21]. Interestingly, the two randomised phase-III trials with bevacizumab did not show the same results. Although both trials demonstrated improved response rates and progression free survival with the addition of bevacizumab to a combination chemotherapy regimen with carboplatin/paclitaxel and cisplatin/gemcitabine, respectively, prolongation of overall survival (OS) has only been demonstrated for the carboplatin/paclitaxel/bevacizumab combination (OS: 12.3 versus 10.3 months; HR 0.80 ; $\mathrm{p}=0.013)[21,22]$. The recently presented AVAPEARL study [23], which was investigating the role of maintenance pemetrexed and bevacizumab after an induction cisplatin/pemetrexed/bevacizumab phase, confirmed the activity of bevacizumab, also in combination with newer chemotherapy agents and demonstrated very promising preliminary survival times. As a 
result of these trials, bevacizumab combined with a platinum-based chemotherapy is a treatment option in patients with performance status $\mathrm{O}^{-1}$ and non-squamous cell histology.

\section{EML4-ALK gene rearrangement}

The most common rearrangement of the ALK gene arises from an inversion in the short arm of chromosome 2 that creates a fusion between the $5^{\prime}$ portion of the EML4 gene and the $3^{\prime}$ portion of ALK. The incidence of the ALK fusion gene, which possesses potent oncogenic activity, is estimated to be $4 \%$ in NSCLC. Patients with ALK gene rearrangements have been reported to exhibit marked sensitivity to crizotinib, a small molecule inhibitor of ALK [24]. A recent retrospective review focusing on the impact of ALK rearrangement on OS in patients treated with crizotinib suggested improved survival compared with that of crizotinib-naive controls [11]. An update of the pivotal phase-I trial with this agent reported on 119 mostly heavily pretreated patients. The median progressionfree survival and the one- and two-year survival were 10 months, $70 \%$ and $55 \%$, respectively, which by far exceeds anything previously observed [25]. Results from randomised crizotinib trials in pretreated or chemotherapy naïve are eagerly awaited.

\section{Other mutations}

In a recently published article the results of mutation testing in $>1,000$ patients with adenocarcimoma of the lung in North America were presented. The incidence of rarer mutations is $2 \%$ for BRAF and $\angle 2 \%$ for $A K T 1$, HER2, MEK, NRAS and PIK 3 CA [26]. In addition, gene amplification of MET was also detected in $<2 \%$. The majority of these genetic alterations are considered to be oncogenic drivers and clinical investigations with corresponding experimental drugs are warranted and ongoing. KRAS mutations are present in approximately one out of five adenocarcinomas and drugs targeting several downstream signalling pathways of RAS such as PIK 3 ACA, ERK, MEK and mTOR or their combinations are currently being explored. While recent research has primarily focused on genetic subtypes of adenocarcinomas, potential targets for squamous cell carcinoma such as focal fibroblast growth factor receptor 1 (FGFR1) amplification (22\%), DDR2 mutations (4\%) and other mutations have been identified and results of early trials are eagerly awaited [27, 28].

\section{Treatment approach}

There are various options of incorporating targeted therapies in the treatment of lung cancer. Currently their indication lies in the palliative treatment of stage-IV non-small cell lung cancer.

The list of targeted agents in lung cancer treatment is growing and currently includes bevacizumab, cetuximab (not approved), erlotinib, gefitinib, crizotinib. For patients with activating EGFR mutations erlotinib or gefitinib is indicated as first-line treatment. Crizotinib is FDA approved in patients that are ALK positive after first line treatment. Cetuximab (not approved) or bevacizumab may also be used in the first line setting in combination with chemotherapy, where cetuximab or bevacizumab should be continued until progression or unacceptable toxicity [29]. Patients with a very poor performance status (PS 3-4) do not benefit from conventional chemotherapy; however, erlotinib or gefitinib may be options in this scenario in case of the presence of an EGFR mutation [29].

As our understanding of the mechanisms is improving, new drugs and tests are being developed and results of closed and ongoing trials are being published.

An example for a recommendation for firstline systemic therapy and the incorporation of targeted drugs into daily routine is displayed in the figure below (adapted from [30]. Recommendations may often need to be adapted to local policies and reimbursement issues should be considered.

Currently there is no role for routine testing of molecular markers in squamous cell carcinoma, but this may soon change as IGF1R amplification [31], DDR $1 / 2$ mutations [32] and other markers are discovered and explored in clinical trials.

\section{Toxicity of targeted therapies}

EGFR inhibitors, although generally well tolerated, do have a specific side effect spectrum, the most prominent side effects being cutaneous reactions. Acneiform rashes, mostly in the head and neck region occur in many 


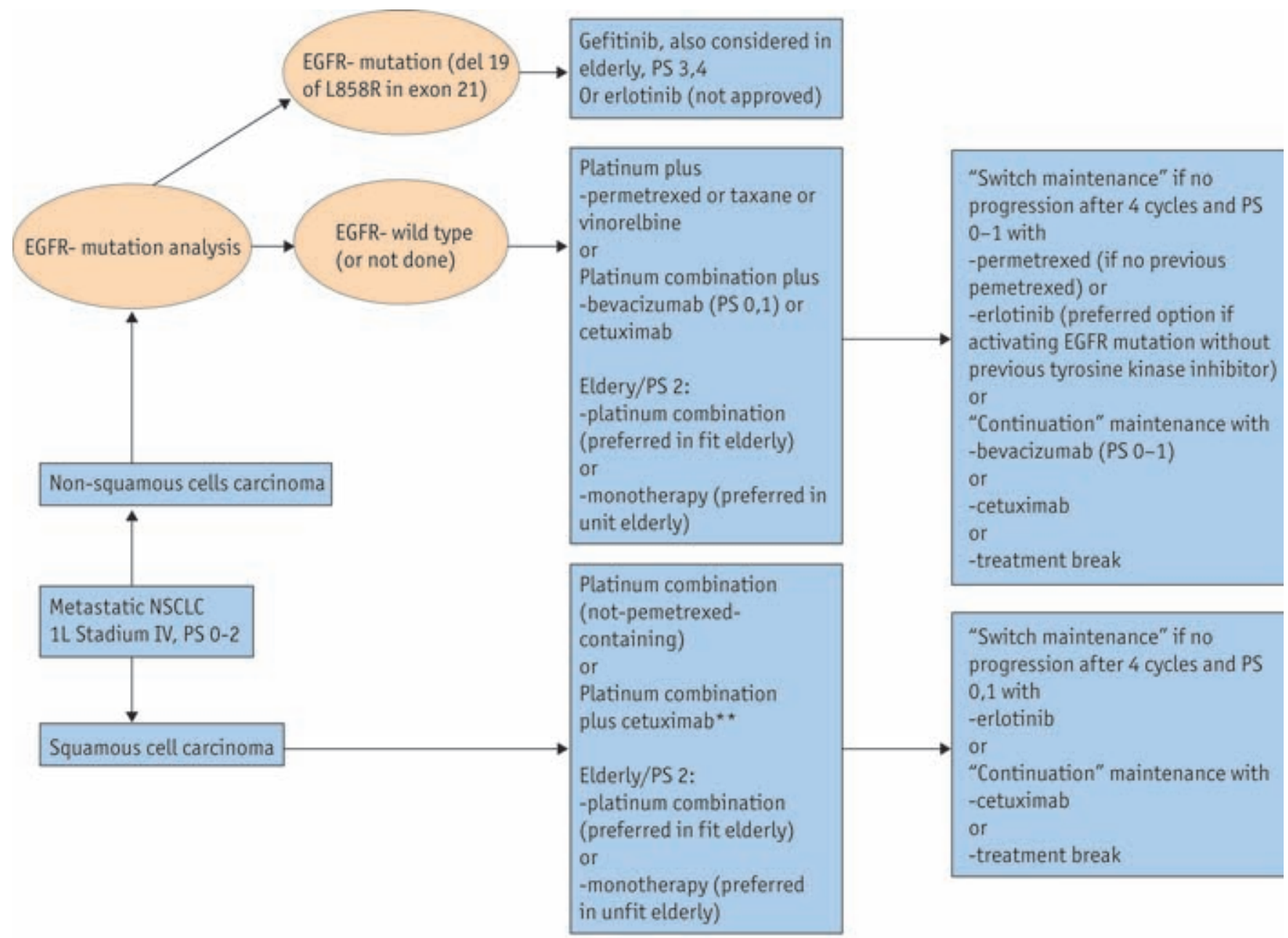

Figure 10

Molecular testing and targeted agents can now be incorporated into first-line treatment of advanced-stage lung cancer patients. EGFR: epidermal growth factor receptor; PS: performance status; NSCLC: non-small cell lung cancer.

patients. Typically this self-limiting side effect can be treated topically and resolve spontaneously in the majority of cases. Treatment should not be interrupted due to this acneiform rash as trials have shown that patients with prominent rashes benefit most from treamtent [19]. Other common side effects include diarrhoea, fatigue and loss of appetite. Antibodies (cetuximab) maybe lead to allergic reactions during infusions, which is why patients are commonly pretreated with antihistamines and steroids.

Infrequent but potentially serious side effects of the VEGF inhibitor bevacizumab include stroke or heart problems, including blood clots, mini-stroke, heart attack and chest pain. High blood pressure is seen in approximately every tenth patient. If the blood pressure can be controlled with medication, treatment can be continued. Due to the risk of serious pulmonary haemorrhage associated with bevacizumab treatment, it should not be used in patients with haemoptysis.

Crizotinib has also been shown to cause treatment related side effects, these included Grade $1 / 2$ vision disorders in approximately two out of three patients, gastrointestinal side effects in every second patients (diarrhoea, vomiting, nausea) and elevated liver enzymes.

\section{Conclusion}

Targeted therapies are increasingly becoming relevant. Their indiscriminate use is associated with higher costs and minimal clinical improvement. However, in selected patients, they may result in a significant benefit. In addition, the toxicity profile is generally favourable when compared to conventional chemotherapy. There are several factors limiting the use of 
targeted agents in lung cancer. Not all substances are approved by regulatory committees (EMEA, FDA) and are therefore not readily available in every country. The higher costs of these treatments can also not be ignored, particularly when given to unselected populations. The efficacy of these drugs is very much dependent on the molecular profiles of tumours and many of the required tests are not readily available in every institution.

Unfortunately, targeted treatments are having their greatest effects only in a small fraction of lung cancer patients who do have the corresponding molecular alteration that responds to the appropriate treatment, leaving the majority of patients $(>85 \%)$ experiencing only marginal benefits. By designing smart clinical trials, which identify subgroups of patients most likely to benefit from targeted treatments early in the course of drug development, we will hopefully be able to increase the use of effective targeted therapies and potentially benefit more lung cancer patients in the future.

\section{REFERENCES}

1. Alberg AJ, Brock MV, Samet JM, et al. Epidemiology of lung cancer: looking to the future. J Clin Oncol 2005; 23: 3175-3185.

2. Jemal A, Bray F, Center MM, et al. Global cancer statistics. CA Cancer J Clin 2011; 61: 69-90.

3. Hyde L, Hyde Cl. Clinical manifestations of lung cancer. Chest 1974; 65: 299-306.

4. Molina JR, Adjei AA, Jett JR. Advances in chemotherapy of non-small cell lung cancer. Chest 2006; 130: 1211-1219.

5. Heyer CM, Reichelt S, Peters SA, et al. Computed tomography-navigated transthoracic core biopsy of pulmonary lesions: which factors affect diagnostic yield and complication rates? Acad Radiol 2008; 15: 1017-1026.

6. Eberhardt R, Anantham D, Ernst A, et al. Multimodality bronchoscopic diagnosis of peripheral lung lesions: a randomized controlled trial. Am J Respir Crit Care Med 2007; 176: 36-41.

7. Yasufuku K, Nakajima T, Motoori K, et al. Comparison of endobronchial ultrasound, positron emission tomography, and CT for lymph node staging of lung cancer. Chest 2006; 130: 710-718.

8. Normanno N, Bianco C, Strizzi L, et al. The ErbB receptors and their ligands in cancer an overview. Curr Drug Targets 2005; 6: 243-257.

9. Connolly DT, Heuvelman DM, Nelson R, et al. Tumor vascular permeability factor stimulates endothelial cell growth and angiogenesis. J Clin Invest 1989; 84: 1470-1478.

10. Choi YL, Soda M, Yamashita Y, et al. EML4-ALK mutations in lung cancer that confer restistance to ALK inhibitors. N Engl J Med 2010; 363: 1734-1739.

11. Shaw AT, Yeap BY, Solomon BJ, et al. Effect of crizotinib on overall survival in patients with advanced non-small-cell lung cancer harbouring ALK gene rearrangement: a retrospective analysis. Lancet Oncol 2011; 12: 1004-1012.

12. Riely G), Politi KA, Miller VA, et al. Update on epidermal growth factor receptor mutation in nonsmall cell lung cancer. Clin Cancer Res 2006; 12: 7232-7241.

13. Kosaka T, Yatabe $\mathrm{Y}$, Endoh $\mathrm{H}$, et al. Analysis of epidermal growth factor receptor gene mutation in patients with non small cell lung cancer and acquired resitatance to gefitinib. Clin Cancer Res 2006; 19: 5764-5769.

14. Engelman JA, Zejnullahu K, Mitsudomi T, et al. MET amplification leads to gefitinib resistance in lung cancer by activating ERBB3 signalling. Science 2007; 5827: 1039-1043.

15. Mok TS, Wu YL, Thongprasert S, et al. Gefitinib or carboplatin-paclitaxel in pulmonary adenocarcinoma. N Engl J Med 2009; 361: 947-957.

16. Maemondo M, Inoue A, Kobayashi K, et al. Gefitinib or chemotherapy for non-small-cell lung cancer with mutated EGFR. N Engl J Med 2010; 362: 2380-2388.

17. Mitsudomi T, Morita S, Yatabe $Y$, et al. Gefitinib versus cisplatin plus docetaxel in patients with nonsmall-cell lung cancer harbouring mutations of the epidermal growth factor receptor (WJTOG 3405): an open label, randomised phase 3 trial. Lancet Oncol 2010; 11: 121-128.

18. Zhou C, Wu YL, Chen G, et al. Erlotinib versus chemotherapy as first-line treatment for patients with advanced EGFR mutation-positive non-small-cell lung cancer (OPTIMAL, CTONG-0802): a multicentre, open-label, randomised, phase-3 study. Lancet Oncol 2011; 12: 735-742.

19. Pirker R, Pereira JR, Szczesna A, et al. FLEX Study Team. Cetuximab plus chemotherapy in patients with advanced non-small-cell lung cancer (FLEX): an open label randomised phase-III trial. Lancet 2009; 373: 1525-1531.

20. Holmes K, Roberts OL, Thomas AM, et al. Vascular endothelial growth factor receptor-2: structure, function, intracellular signalling and therapeutic inhibition. Cell Signal 2007; 19: 2003-2012.

21. Sandler A, Gray R, Perry MC, et al. Paclitaxelcarboplatin alone or with bevacizumab for non-smallcell lung cancer. N Engl J Med 2006; 355: 2542-2550.

22. Reck M, von Pawel J, Zatloukal P, et al. Phase III trial of cisplatin plus gemcitabine with either placebo or bevacizumab as first-line therapy for nonsquamous non-small-cell lung cancer: AVAil. J Clin Oncol 2009; 27: 1227-1223.

23. Barlesi F, de Castro J, Dvornichenko V, et al. AVAPERL: Final efficacy outcomes after 1st line bevcisplatin for pts with advanced non-squamous nonsmall cell lung cancer randomized to continuous maintenance with bevacizumab (bev) or bev+premetrexed after 1st-line bev-cisplatin-premetrexed treatment. 6th ECCO/36th ESMO European Cancer Congress. Abstract 34LBA.

24. Kwak EL, Bang YJ, Camidge DR, et al. Anaplastic lymphoma kinase inhibition in non-small-cell lung cancer. N Engl J Med 2010; 363: 1693-1703.

25. Camidge DR, Bang Y, Kwak EL, et al. Progression-free survival (PFS) from a phase I study of crizotinib 
(PF-02341066) in patients with ALK-positive nonsmall cell lung cancer (NSCLC). J Clin Oncol 2011; 29: suppl. abstr 2501.

26. Chaft JE, Arcila ME, Paik PK, et al. Coexistence of $\mathrm{PIK}_{3} \mathrm{CA}$ and other oncogene mutations in lung adenocarcinoma - rationale for comprehensive mutation profiling. Mol Cancer Ther, 2011; 1. [Epub ahead of print].

27. Weiss J, Sos ML, Seidel D, et al. Frequent and focal FGFR1 amplification associates with therapeutically tractable FGFR1 dependency in squamous cell lung cancer. Sci Transl Med 2010; 2: 62-93.

28. Hammerman PS, Sos ML, Ramos $\mathrm{AH}$, et al. Mutations in the DDR2 kinase gene identify a novel therapeutic target in squamous cell lung cancer. Cancer Discovery 2011; 1: 78-89.

29. National Comprehensive Cancer Network. NCCN Clinical Practice Guidelines in Oncology; Non-Small Cell Lung Cancer. Version 2.2012.

30. Frueh M. The search for improved systemic therapy of non-small cell lung cancer-what are today's options? Lung Cancer 2011; 72: 265-270.

31. Nakagawa M, Uramoto H, Oka S, et al. Clinical significance of IGFıR expression in non-small-cell lung cancer. Clin Lung Cancer, 2011. [Epub ahead of print].

32. Yang SH, Baek HA, Lee HJ, et al. Discoidin domain receptor 1 is associated with poor prognosis of nonsmall cell lung carcinomas. Oncol Rep 2010; 24: 311-319. 